\title{
Anxiety Network of Brain Function in Patients with Acute Cerebral Infarction
}

\author{
Huanyin Li1, Huiwen Gui ${ }^{1}$, Ye Yao ${ }^{2 *}$, Jixian Lin ${ }^{1,3^{*}}$ \\ ${ }^{1}$ Department of Neurology, Minhang Branch, Zhongshan Hospital, Fudan University, Shanghai, China \\ ${ }^{2}$ Department of Biostatistics, School of Public Health, Fudan University, Shanghai, China \\ ${ }^{3}$ School of Information Science and Technology, Fudan University, Shanghai, China \\ Email: *yyao@fudan.edu.cn, *jixianlin@hotmail.com
}

How to cite this paper: Li, H.Y., Gui, H.W., Yao, Y. and Lin, J.X. (2021) Anxiety Network of Brain Function in Patients with Acute Cerebral Infarction. Health, 13, 777-787.

https://doi.org/10.4236/health.2021.137060

Received: June 25, 2021

Accepted: July 23, 2021

Published: July 26, 2021

Copyright $\odot 2021$ by author(s) and Scientific Research Publishing Inc. This work is licensed under the Creative Commons Attribution International License (CC BY 4.0).

http://creativecommons.org/licenses/by/4.0/

\begin{abstract}
Objective: To explore the characteristics of brain functional network with anxiety in patients with acute cerebral infarction. Methods: A total of 39 patients with acute cerebral infarction by cranial magnetic resonance examination were included, and all the patients were scored by the Hamilton Anxiety Scale. The anxiety scale is scored by a professional psychiatrist. There are a total of 14 items, including anxiety, nervousness, fear, insomnia, cognitive function, depressed mood, somatic anxiety, sensory system, etc. The total score $\geq 29$ points may be severe; $\geq 21$ points, there must be obvious; $\geq 14$ points, there must be anxiety; a score of more than 7 may indicate anxiety. If the score is less than 7, there are no anxiety symptoms. All patients within 24 to 72 hours, complete the head examination magnetic resonance, computerized calculation of the DWI sequence images, according to the results of the calculation to superimpose the image of the lesion, image reconstruction in space, and carry out Binarization, defining the value of lesions as 1 , and the value of non as 0 . All lesions are superimposed into one image and integrated. The relationship between the lesions in this superimposed image and anxiety after cerebral infarction was analyzed. Results: The lesions were basically concentrated around the lateral ventricle, and they were mainly concentrated around the lateral ventricle. Conclusion: Patients with acute cerebral infarction in the lateral ventricle or basal ganglia are more prone to post-stroke anxiety. This has a certain evaluation value for the prognosis of future cerebral infarction, and has a certain understanding of the exploration of complications, and has a certain understanding of the exploration of complications.
\end{abstract}

\section{Keywords}

Acute Cerebral, Infarction, Anxiety, MRI, Brain Functional Network 


\section{Introduction}

Anxiety disorder is a common clinical condition. Also known as anxious neurosis, neurosis is one of the most common diseases in the category of neurosis, characterized by the experience of anxious emotions [1]. It can be divided into chronic anxiety (or generalized anxiety) and acute anxiety (or panic attack). The main performance is: there is no clear objective object of nervous worry, fidgeting, and autonomic nervous dysfunction symptoms, such as palpitations, hand shaking, sweating, frequency of urine, etc. Note the distinction between normal anxiety, which may be pathological, if its severity is significantly inconsistent with objective facts or situations, or if its duration is too long. We commonly use the Hamilton Anxiety Scale to evaluate the patient's anxiety. The Hamilton Anxiety Scale includes anxiety; Stress; Fear; Insomnia; Cognitive function; Inability to concentrate, poor memory; Depressive mood; Sensory system symptoms; Cardiovascular system symptoms; Respiratory symptoms; Gastrointestinal symptoms; Reproductive and urinary symptoms; Autonomic nervous system symptoms; How you behave when you're talking to someone. After acute cerebral infarction, anxiety and depression are easy to be complicated; this is one of the most common complications of cerebral infarction [2]. Most of them occur about 2 months to 1 year after cerebral infarction, which not only aggravates physical symptoms and mental pain, but also delays the rehabilitation process of cerebral infarction [3]. At present, the related etiology and mechanism of anxiety and depression are not very clear; therefore, the mechanism of anxiety and depression after acute cerebral infarction is not very clear. At the same time, because the reaction of anxiety and depression occurs very quietly, it is not easy to detect. In some patients, the symptoms of anxiety and depression cannot be detected due to the language barrier, which is often not known until after the occurrence of an accident. Therefore, the quality of life and prognosis of patients are seriously affected, and for them the early intervention and treatment should be carried out. The anxiety depression after acute cerebral infarction includes two kinds of pathogenesis theory [4]: the first is cerebral infarction lesions caused by single amine neurotransmitter levels or its receptor function decline and anxiety depression symptoms, the second is that anxiety and depression after cerebral infarction is a disorder of hypothalamus-pituitary-adrenal axis caused by brain damage, which leads to regulatory disorders. But at present for acute cerebral infarction after the reactive mechanism of anxiety research less, there are also people who believe that anxiety depression after acute cerebral infarction is related to the site of cerebral infarction. Nobuhara et al. believed that patients with damaged neural microstructures in the frontal and temporal lobes are more prone to anxiety and depression symptoms [5]. Vataja et al. found that patients with cerebral infarction in the subcortical pathway of the frontal lobe are more likely to develop anxiety and depression after cerebral infarction [6]. However, some studies also believed that the stroke site was not related to the occurrence of anxiety and depression [7]. Therefore, this study intends to ex- 
plore the possible mechanism and loop of anxiety in patients after acute cerebral infarction, so as to guide the treatment of anxiety after infarction and early intervention of anxiety, so as to improve the quality of life of patients after infarction.

\section{Objects and Methods}

\subsection{Objects}

Research object collected from January 2019 to June 2019 nerve internal medicine stroke unit of patients with acute cerebral infarction, the set of standard: in accordance with Chinese guide 2018 diagnostic criteria of diagnosis and treatment of acute ischemic stroke for patients with acute cerebral infarction [8], at the same time, 24 to 48 hours after admission professional perfect head MRI image doctor, clear with acute cerebral infarction lesions, through two psychiatrists in professional Hamilton Anxiety Scale (HAMD14-item version) do [9] anxiety score, a total of 29 or more points, for serious anxiety; $\geq 21$ points, obvious anxiety; $\geq 14$ points, anxiety; Over 7, anxiety; If the score is less than 7, there are no anxiety symptoms. Exclusion criteria: 1) patients with a history of head trauma; 2) a history of brain parenchymal disease, seizure or syncope; 3 ) a history of substance abuse or alcohol abuse; 4) a history of serious systemic disease, including cardiac, hepatic and renal insufficiency; 5) a history of pregnancy or lactation; 6) a history of mental illness. Screening out of a total of 39 cases, the entire group, all cases after cranial MRI examination, clear of acute cerebral infarction, including the brain stem infarction in 5 cases, 13 cases of lobes, basal ganglia and lateral ventricle infarction, 21 cases, all patients with image data, the computer $2 \mathrm{~d}$ cutting, overlapping imaging, binarization processing, displayed on the space, expressed as a value of 1 lesion, show with warm color, no pathological changes with the number 0 say, show with colorless in space.

\subsection{Research Methods}

\subsubsection{Clinical Evaluation}

The clinical data of the subjects included age, sex, education level, and history of hypertension, diabetes, Hyperlipidemia, coronary heart disease, and transient ischemic attack, and Stroke Scale (NIHSS) from the National Institutes of Health and TOAST classification of the subjects were used for clinical assessment.

\subsubsection{MRI Data Collection}

Head MRI data were collected by imaging physicians, and 3.0T MAGNETIC resonance imaging system (Siemens, Verio) produced in Germany was used. The scanning adopts gradient echo-echo plane imaging technique, scanning parameters: repeat time/echo time is $2000 \mathrm{~ms} / 35 \mathrm{~ms}$, turning Angle is $90^{\circ}$, matrix is $64 \times 64$, visual field is $240 \mathrm{~mm} \times 240 \mathrm{~mm}$, layer thickness/layer interval is 4 $\mathrm{mm} / 0.8 \mathrm{~mm}$. Before the scan, explain the scanning process and precautions to the subjects. During the scan, the subjects should keep quiet, close their eyes and keep their eyes fixed, and try to avoid head and body movement. 


\subsubsection{Method}

Patients were tested within 3 - 4 weeks of their first stroke using a 14-item version of the Hamilton Anxiety Scale (HAMD). The total score $\geq 29$ is defined as severe anxiety; $\geq 21$ points, obvious anxiety; $\geq 14$ points, anxiety; over 7 , anxiety; if the score is less than 7 , there are no symptoms of anxiety.

\subsubsection{Data Processing}

The area of each ischemic site of acute cerebral infarction was calculated by DWI sequence on MAGNETIC resonance imaging (MRI), and the Hamilton anxiety scale score was calculated using Matlab (R2012b) software Parametric Mapping 8. The relationship between acute cerebral infarction and Hamilton Anxiety Scale scores was completed by MRIcron 4.8.2014 mapping Toolkit (University of South Carolina, USA). For acute cerebral infarction lesions area, Hamilton anxiety scale scores of numerical analyzed, there is damage to the corresponding is the part of the acute cerebral infarction, select threshold (cluster voxel size 100 individual element or higher) after $t$ value of each component diagram, expressed as a value of 1 , done through graphics toolkit, the above steps for each component for the parts without damage, is corresponding to the other without cerebral infarction area, with the number 0 , overlapping in the two-dimensional space, finally it is concluded that under the resting state of brain functional network map, which shows that the network between the two groups in different brain regions, corresponding. The Hamilton anxiety score corresponding to the site of acute cerebral infarction is high, indicating a high incidence of anxiety, which can be used for early intervention of anxiety after acute cerebral infarction.

\subsubsection{Statistical Methods}

SPSS 16.0 was used for Pearson correlation analysis of age, sex, smoking, diabetes, hyperlipidemia and other conditions of the enrolled patients, and $\mathrm{P}<$ 0.05 was used as the test level. Independent sample $T$ test was used for measurement data, chi-square test was used for counting data, and $\mathrm{P} \leq 0.05$ was considered statistically significant.

\section{Results}

General clinical characteristics comparison: total income of 62 cases of acute cerebral infarction patients, with Hamilton Depression Scale scores, the final diagnosis of 39 patients with anxiety disorders, with age, sex, smoking, diabetes, dyslipidemia, hypertension (including systolic and diastolic blood pressure), TOAST classification, relationship between NIHSS scores and Hamilton Anxiety Scale, the relationship between the Pearson correlation analysis, according to the results found that HAMD scores and gender into positive correlation $(\mathrm{P}=0.05)$, and anxiety into positive correlation $(\mathrm{P}=0.04)$, no more see significant correlation (see Table 1). According to the Hamilton score, there were 9 patients with mild anxiety disorder, 26 patients with moderate anxiety disorder, and 4 patients with severe anxiety disorder (Table 2). Among them, there were no statistically 
Table 1. The relationship between anxiety in each stroke site.

\begin{tabular}{ccccccccc}
\hline & \multicolumn{2}{c}{ Sex } & \multicolumn{3}{c}{ Stroke site } & \multicolumn{2}{c}{ Brain parts } \\
\cline { 2 - 9 } Number of anxiety disorders & Male & female & $\begin{array}{c}\text { Brain } \\
\text { stem }\end{array}$ & $\begin{array}{c}\text { brain } \\
\text { lobe }\end{array}$ & $\begin{array}{c}\text { lateral } \\
\text { ventricle }\end{array}$ & Left & Right \\
\hline $\begin{array}{c}\text { Mild to moderate } \\
\text { severe }\end{array}$ & 15 & 16 & 19 & 5 & 11 & 19 & 15 & 15 \\
P & 1 & 3 & 0 & 1 & 3 & 2 & 2 \\
\hline
\end{tabular}

Table 2. Anxiety disorders and comorbidities.

\begin{tabular}{cccccc}
\hline $\begin{array}{c}\text { Number of anxiety } \\
\text { disorders }\end{array}$ & $\mathrm{N}$ & hypertension & diabete & hyperlipidemia & $\begin{array}{c}\text { coronary heart } \\
\text { disease }\end{array}$ \\
\hline Mild to moderate & 35 & $16(45.7 \%)$ & $20(57.1 \%)$ & $15(42.9 \%)$ & $8(22.9 \%)$ \\
severe & 4 & $3(75.0 \%)$ & $2(50.0 \%)$ & $3(75.0 \%)$ & $2(50.0 \%)$ \\
P & & & 0.836 & \\
\hline
\end{tabular}

significant differences between the number of anxiety disorders in males and females, or between stroke sites, $P \geq 0.05$ (Table 1).

From the table, comparison of vascular risk factors between the two groups, the comparison of hypertension, diabetes, hyperlipidemia and coronary heart disease between the mild to moderate anxiety group and the severe anxiety group was $\mathrm{P}>0.05$, indicating that there was no statistically significant difference in vascular risk factors between the two groups (Table 2).

Analysis of demographic data of acute cerebral infarction patients with anxiety (Figure 1): the average age of the participants was $65.97 \pm 12.39$ years old, including 20 males and 19 females, among whom gender and anxiety may be related, $\mathrm{P}=0.05,12$ patients with smoking history and 27 patients with no smoking history, $\mathrm{P}=0.04$, indicating that smoking is related to anxiety. There were 8 diabetic patients, 12 with abnormal lipid metabolism, and 21 with hypertension, with an average systolic blood pressure of $138.38 \pm$ 17.97 MMHG. The mean diastolic blood pressure was $78.13 \pm 9.28 \mathrm{HHMG}$, and the mean heart rate was $76.54 \pm 5.99$ times/min. Among the five TOAST classifications, 21 cases are of great atherosclerosis, 2 cases of cardiology, 11 cases of small artery occlusion, 2 cases of clinically rare causes, and 3 cases of unknown causes. The average NIHSS score was $3.54 \pm 3.12$, the average LDL was $3.03 \pm 0.88 \mathrm{mmol} / \mathrm{L}$, and the average homocysteine level was $17.33 \pm 14.13$. The mean hBAlc was $6.54 \% \pm 1.62 \%$, the mean blood glucose was $5.87 \pm 2.12 \mathrm{mmol} / \mathrm{L}$, and the blood creatinine was 75.62 $\pm 16.87 \mathrm{umol} / \mathrm{L}$, urea nitrogen $4.85 \pm 1.26 \mathrm{mmol} / \mathrm{L}$, uric acid $314.95 \pm 93.52$ umol/L, platelet count $230.23 \pm 61.53 \times 10^{9}$.

Analysis of Hamilton Anxiety Scale: The average score was $6.23 \pm 4.6$, among which, the score was $0.69 \pm 0.66$ for anxiety, $0.15 \pm 0.43$ for nervousness, $0.13 \pm$ 0.41 for fear, $0.79 \pm 0.73$ for insomnia, $1.03 \pm 0.71$ for cognitive impairment, 0.67 \pm 0.66 for depressive mood, $0.49 \pm 0.56$ for muscular symptoms, $0.26 \pm 0.50$ for 


\begin{tabular}{|c|c|c|c|}
\hline \multicolumn{2}{|c|}{$\begin{array}{l}\text { Demographic, Clinical } \\
\text { and Anxiety Data }\end{array}$} & Patients with Stroke & Relationship with Anxiety \\
\hline \multicolumn{2}{|l|}{ Age (years) } & $65.97 \pm 12.39,44.00-92.00$ & $t=-2.00, p=0.05$ \\
\hline \multicolumn{2}{|c|}{ Gender male/female } & $20 / 19$ & $t=-2.00, p=0.05$ \\
\hline \multicolumn{2}{|l|}{ Smoking yes/no } & $12 / 27$ & $t=-2.11, p=0.04$ \\
\hline \multicolumn{2}{|l|}{ Diabetes yes/no } & $8 / 31$ & $t=0.79, p=0.44$ \\
\hline \multicolumn{2}{|c|}{ Lipid Metabolism Disorders yes/no } & $12 / 27$ & $t=0.09, p=0.93$ \\
\hline \multicolumn{2}{|c|}{ Hypertension yes/no } & $21 / 18$ & $t=0.09, p=0.93$ \\
\hline \multicolumn{2}{|c|}{ Systolic Blood Pressure (mmHg) } & $138.38 \pm 17.97,104.00-200.00$ & $r=-0.02, p=0.90$ \\
\hline \multicolumn{2}{|c|}{ Diastolic Blood Pressure (mmHg) } & $78.13 \pm 9.28,60.00-100.00$ & $r=-0.15, p=0.36$ \\
\hline \multicolumn{2}{|l|}{ Pulse (bpm) } & $76.54 \pm 5.99,60.00-90.00$ & $r=-0.12, p=0.45$ \\
\hline \multicolumn{2}{|c|}{ TOAST Classificationt } & $21 / 2 / 11 / 2 / 3$ & $F=0.67, p=0.61$ \\
\hline \multicolumn{2}{|l|}{ NIH Stroke Scale } & $3.54 \pm 3.120 .00-14.00$ & $r=0.08, p=0.65$ \\
\hline \multicolumn{2}{|c|}{ Low Density Lipoprotein (mmol/L) } & $3.03 \pm 0.88,1.79-5.48$ & $r=-0.06, p=0.73$ \\
\hline \multicolumn{2}{|c|}{ Homocysteine ( $\mu \mathrm{mol} / \mathrm{L})$} & $17.33 \pm 14.13,8.60-82.40$ & $r=-0.01, p=0.96$ \\
\hline \multicolumn{2}{|c|}{ Glycated Hemoglobin(\%) } & $6.54 \pm 1.62,5.10-12.80$ & $r=0.12, p=0.48$ \\
\hline \multicolumn{2}{|c|}{ Fasting Blood Glucose ( $\mathrm{mmol} / \mathrm{L})$} & $5.87 \pm 2.12,4.10-14.50$ & $r=0.10, p=0.53$ \\
\hline \multicolumn{2}{|c|}{ Serum Creatinine ( $\mu \mathrm{mol} / \mathrm{L})$} & $75.62 \pm 16.87,48.00-119.00$ & $r=-0.07, p=0.66$ \\
\hline \multicolumn{2}{|c|}{ Serum Urea Nitrogen $(\mu \mathrm{mol} / \mathrm{L})$} & $4.85 \pm 1.26,2.40-7.80$ & $r=0.17, p=0.31$ \\
\hline \multicolumn{2}{|l|}{ Uric Acid ( $\mu \mathrm{mol} / \mathrm{L})$} & $314.95 \pm 93.52,124.00-485.00$ & $r=0.07, p=0.68$ \\
\hline \multicolumn{2}{|l|}{ Platelet (x109/L) } & $230.23 \pm 61.53,107.00-385.00$ & $r=-0.19, p=0.24$ \\
\hline \multicolumn{4}{|c|}{ Hamilton Anxiety Rating Scale (HAM-A) } \\
\hline Total Score & $6.23 \pm 4.60,0.00-22.00$ & & \\
\hline Anxious Mood & $0.69 \pm 0.66,0.00-3.00$ & Somatic (Sensory) & $0.26 \pm 0.50,0.00-2.00$ \\
\hline Tension & $0.15 \pm 0.43,0.00-2.00$ & Cardiovascular Symptoms & $0.46 \pm 0.64,0.00-2.00$ \\
\hline Fears & $0.13 \pm 0.41,0.00-2.00$ & Respiratory Symptoms & $0.62 \pm 0.59,0.00-2.00$ \\
\hline Insomnia & $0.79 \pm 0.73,0.00-2.00$ & Gastrointestinal Symptoms & $0.13 \pm 0.34,0.00-1.00$ \\
\hline Intellectual & $1.03 \pm 0.71,0.00-2.00$ & Genitourinary Symptoms & $0.00 \pm 0.00,0.00-0.00$ \\
\hline Depressed Mood & $0.67 \pm 0.66,0.00-2.00$ & Autonomic Symptoms & $0.18 \pm 0.51,0.00-2.00$ \\
\hline Somatic (Muscular & $r^{\prime} 0.49 \pm 0.56,0.00-2.00$ & Behavior at Interview & $0.74 \pm 0.68,0.00-3.00$ \\
\hline $\begin{array}{l}{ }^{*} \text { Continuous dat } \\
\text { relationships bas } \\
\text { anviety relationsł } \\
\text { clssifications: (1) I } \\
\text { of otherdetermin }\end{array}$ & $\begin{array}{l}\text { a are shown as mean } \pm \\
\text { ed on Pearson coelation } \\
\text { hips based on two sampl } \\
\text { large-artery atherosclero } \\
\text { led etiology and (5) strok }\end{array}$ & $\begin{array}{l}\text { SD, minimum and maximum valu } \\
\text { coefficient }(r \& p) \text {.Categorical da } \\
\text { e t test }(t \& p) \text { and ANOVA test } \\
\text { sis, (2) cardioembolism,(3) small } \\
\text { e of undetermined etiology. }\end{array}$ & $\begin{array}{l}\text { Jes in patients with ansiety } \\
\text { ta are represented with } \\
\text { F \& p).TOAST refers to fve } \\
\text { l-vessel occlusion,(4) stroke }\end{array}$ \\
\hline
\end{tabular}

Figure 1. Demographic, clinical and anxiety data of patients with stroke.

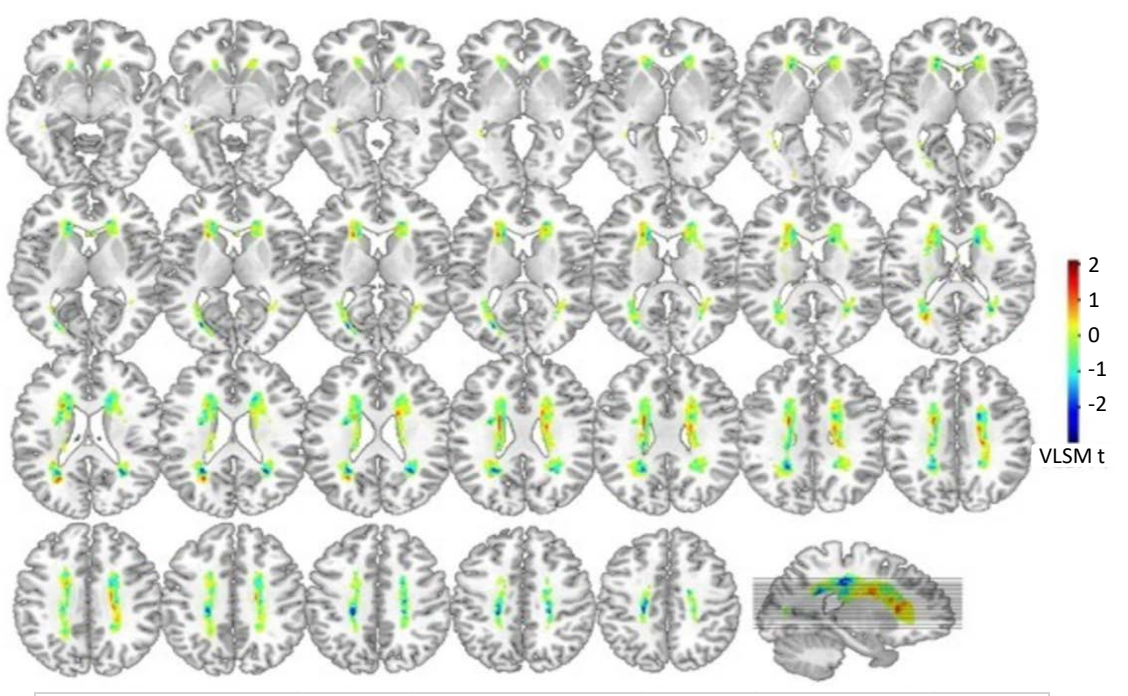

Figure 2 Voxel-based Lesion-Symptom Mapping for Anxiety SymptomsRepresentative slices from VLSM maps computed for anxiety symptoms of 39 patients with stroke. These maps arecolorized depictions of t-test results evaluating patient performance on a voxel-byvoxel basis. The warmer the coloris, patients with lesions on the corresponding voxel hold higher Hamilton anxiety rating scale scores. Age, genderand smoking effects have been removed.

Figure 2. Lesion-symptom mapping for anxiety symptoms. 
sensory system, $0.46 \pm 0.64$ for cardiovascular system, $0.62 \pm 0.59$ for respiratory system. Gastrointestinal symptoms score was $0.13 \pm 0.34$, urinary system score was 0 , autonomic nervous system score was $0.18 \pm 0.51$, and behavioral performance score was $0.74 \pm 0.68$.

After all voxels were combined in two-dimensional space, the brain functional network map was formed (Figure 2). 39 cases of anxiety symptoms in patients with cerebral infarction VLSM images, the representative image, computing from VLSM in 39 patients with symptoms of anxiety in the patients with acute cerebral infarction was calculated, the application of $\mathrm{T}$ value, for the color display on the Internet, to assess the patient's performance in the form of pursuit of individual element, color the warmer part of the corresponding pathological change, the more obvious on the voxel, the corresponding Hamilton anxiety rating scale, the higher the grade, at the same time, age, sex, and smoking factors have been eliminated.

\section{Discussion and Conclusion}

Cerebral infarction is a common and frequently occurring disease that seriously endangers human health and can easily cause various physical and cognitive dysfunctions [10]; there will also be cognitive dysfunction after stroke, including anxiety and depression. Depression is called post-stroke depression (PSD). There has been much discussion for poststroke depression and anxiety for the discussion of the relatively few, so this article mainly discusses the possible mechanism of the occurrence of anxiety. After acute cerebral infarction, patients will have abnormal speech function, limb paralysis, tinnitus, headache, dizziness and other discomforts. Some patients will have dysphagia, nausea, coma and other serious conditions. This kind of patients with clinical manifestations will cause concern about their own situation, thus appeared restlessness, irritability, anxiety, as the extension of time, depression, approximately $60 \%$ of patients will experience post-stroke anxiety and depression [11]. Post-stroke anxiety and depression can easily affect the quality of life of patients and increase the burden on family and society [12]. At present, the mechanism of post-stroke anxiety and depression is not clear, and most of them prefer the psychosocial medical model. It has been thought that DLPFC participates in the regulation of negative emotions such as anxiety and depression [13] [14] [15], as well as cognitive executive functions such as goal-oriented planning and cognitive reevaluation [16]. So is post-stroke cognitive dysfunction associated with intracranial dorsolateral superior frontal gyrus?

There's no evidence for this, and it's generally believed that people with anxiety and depression have organic changes in brain tissue. It is mainly targeted at the neural loop of the limbic-cortical-striatal-pallidal-thalamic system (LCSPT) [17]. Therefore, the basal ganglia region is an important part of the loop, among which the putamen, pallium, caudate nucleus, dorsal thalamus, etc., are important structures within the loop. The striatum in the basal ganglia region is di- 
vided into dorsal and ventral parts. The striatum of the basal ganglia is divided into dorsal and ventral sections. The aminergic cells emanating from the back are 5-HT cell clusters and NE cell clusters, which are respectively projected to the somatosensory motor cortex and the frontal dorsal medial supplementary motor area. The lateral amine energy comes from the dorsal raphe nucleus and locus coeruleus, passes through the dorsal medial nucleus of the thalamus, the raphe nucleus, and radiates to the prefrontal cortex and cingulate cortex. As a result, the basal ganglia region is an important part of the limbic system; moreover, previous research also confirmed that the basal ganglia infarction can produce anxiety and depressive symptoms, but has not explored the loop. After acute cerebral infarction, the brain structure is destroyed, and a large number of complex nerve nuclei gather in the medulla oblongata reticular structure, thalamus, hypothalamus, optic chiasm and other circuits. After the damage of this circuit, the 5-HT and norepinephrine are low, which is easy to lead to the occurrence of post-stroke anxiety and depression. Therefore, it is of great theoretical value and clinical significance to understand the damage of nerve fibers in the basal ganglia region and the occurrence of post-stroke anxiety and depression.

The occurrence of anxiety and depression after cerebral infarction, is not caused by single factors, is the biological-psychology-social factors together with the results, although the real cause of anxiety and depression pathway is not very clear, after stroke of indifference and neural network (connect the anterior cingulate, frontal lobe, caudate nucleus, globus pallidus, thalamus dorsolateral nucleus) related to the damage, may be by destroying nerve conduction pathway, interfere with the neurotransmitter passing and implementation. In patients with post-infarction anxiety and depression, infarcts were more likely to occur in the posterior and basal ganglia of the radio-corona, especially in the caudate nucleus and globus pallidus of the left hemisphere, than in patients without post-infarction anxiety and depression. Compared with patients without anxiety and depression whose infarction foci were located in the basal ganglia, patients with anxiety and depression had a larger range of infarction foci in the basal ganglia [18], and anxiety was most likely to occur around 2 weeks after infarction, with an incidence of up to $29.5 \%$ [2]. Interestingly, the occurrence of depression after cerebral infarction does not necessarily predict the occurrence of post-stroke depression, while the occurrence of anxiety does better predict post-stroke anxiety [19]. Logistic regression analysis showed that: the involvement of globus pallidus anxiety depression after cerebral infarction infarction is a magnetic resonance can be observed that independent high risk factors, at present, most scholars believe that the left hemisphere damage associated with anxiety, depression, to the left frontal lobe and left basal ganglia injury patients [20] higher incidence of anxiety and depression, which related to the anterior cortex mechanism. A number of studies have confirmed that damage to some nuclei in the basal ganglia region can lead to anxiety and depression symptoms. In addition, in addition to the predictive effect of a single infarct on post-infarction 
anxiety and depression, the vascular effect accumulated by repeated lacunar infarcts in the basal ganglia region can also increase the risk of post-stroke anxiety and depression, and it is also easy to cause anxiety due to insufficient cerebral blood perfusion and impaired cerebral blood flow regulation [21]. The results of this study are consistent with previous research results, which further prove the important influence of the basal region in the important link of LCSPT on PSD. It indicates that the basal region, a high incidence area of stroke, is also a sensitive area for PSD, which indirectly proves the scientific nature of LCSPT loop theory.

The diagnosis of post-infarction anxiety and depression is complex, without an absolute standard, and requires multiple considerations. At the same time, due to the limitation of related disciplines, imaging major, neurology major and psychiatry major need to work together. This case study also has certain limitations, because the number of samples is relatively small. At the same time, due to the bias of MRI image collection and data storage, there is a bias in voxel synthesis, resulting in the bias of the study results. Next, we will further expand the sample size and conduct further research in the neuropsychological aspect to understand the pathogenesis of post-stroke depression.

\section{Conflicts of Interest}

The authors declare no conflicts of interest regarding the publication of this paper.

\section{References}

[1] Moser, D.K., Riegel, B., McKinley, S., Doering, L.V., An, K. and Sheahan, S. (2007) Impact of Anxiety and Perceived Control on In-Hospital Complications after Acute Myocardial Infarction. Psychosomatic Medicine, 69, 10-16. https://doi.org/10.1097/01.psy.0000245868.43447.d8

[2] Rafsten, L., Danielsson, A. and Sunnerhagen, K.S. (2018) Anxiety after Stroke: A Systematic Review and Meta-Analysis. Journal of Rehabilitation Medicine, 50, 769-778. https://doi.org/10.2340/16501977-2384

[3] Lincoln, N.B., Brinkmann, N., Cunningham, S., et al. (2013) Anxiety and Depression after Stroke: A 5 Year Follow-Up. Disability and Rehabilitation, 35, 140-145. https://doi.org/10.3109/09638288.2012.691939

[4] Loubinoux, I., Kronenberg, G., Endres, M., et al. (2012) Post-Stroke Depression: Mechanisms, Translation and Therapy. Journal of Cellular and Molecular Medicine, 16, 1961-1969. https://doi.org/10.1111/j.1582-4934.2012.01555.x

[5] Nobuhara, K., Okugawa, G., Sugimoto, T., et al. (2006) Frontal White Matter Anisotropy and Symptom Severity of Late-Life Depression: A Magnetic Resonance Diffusion Tensor Imaging Study. Journal of Neurology, Neurosurgery \& Psychiatry, 77, 120-122. https://doi.org/10.1136/jnnp.2004.055129

[6] Vataja, R., Leppävuori, A., Pohjasvaara, T., et al. (2004) Poststroke Depression and Lesion Location Revisited. The Journal of Neuropsychiatry and Clinical Neurosciences, 16, 156-162. https://doi.org/10.1176/jnp.16.2.156

[7] Wongwandee, M., Tangwongchai, S. and Phanthumchinda, K. (2012) Relationship 
between Poststroke Depression and Ischemic Lesion Location. Journal of the Medical Association of Thailand, 95, 330-336.

[8] Chinese Medical Association Neurology Branch and Chinese Medical Association Neurology Branch Cerebrovascular Disease Group (2018) Chinese Acute Ischemic Stroke Diagnosis and Treatment Guide 2018. Chinese Journal of Neurology, 51, 666-682.

[9] Tang, W.K., Lau, C.G., Mok, V., Ungvari, G.S. and Wong, K.S. (2013) Impact of Anxiety on Health-Related Quality of Life after Stroke: A Cross-Sectional Study. Archives of Physical Medicine and Rehabilitation, 94, 2535-2541. https://doi.org/10.1016/j.apmr.2013.07.012

[10] Liu, Z., Cai, Y., Zhang, X., Zhu, Z. and He, J. (2018) High Serum Levels of Malondialdehyde and Antioxidant Enzymes Are Associated with Post-Stroke Anxiety. Neurological Sciences, 39, 999-1007. https://doi.org/10.1007/s10072-018-3287-4

[11] Almhdawi, K.A., Alazrai, A., Kanaan, S., et al. (2020) Post-Stroke Depression, Anxiety, and Stress Symptoms and Their Associated Factors: A Cross-Sectional Study. Neuropsychological Rehabilitation, 31, 1091-1104.

[12] Sanner, B.J.E., Casameni, M.T., Cai, C., et al. (2020) A Retrospective Study to Identify Novel Factors Associated with Post-stroke Anxiety. Journal of Stroke and Cerebrovascular Diseases, 29, Article ID: 104582.

https://doi.org/10.1016/j.jstrokecerebrovasdis.2019.104582

[13] Verhoeven, C.L., Post, M.W., Schiemanck, S.K., van Zandvoort, M.J., Vrancken, P.H. and van Heugten, C.M. (2011) Is Cognitive Functioning 1 Year Poststroke Related to Quality of Life Domain. Journal of Stroke and Cerebrovascular Diseases, 20, 450-458. https://doi.org/10.1016/j.jstrokecerebrovasdis.2010.02.018

[14] Park, J. and Moghaddam, B. (2017) Impact of Anxiety on Prefrontal Cortex Encoding of Cognitive Flexibility. Neuroscience, 345, 193-202.

https://doi.org/10.1016/j.neuroscience.2016.06.013

[15] Pati, S., Sood, A., Mukhopadhyay, S. and Vaidya, V.A. (2018) Acute Pharmacogenetic Activation of Medial Prefrontal Cortex Excitatory Neurons Regulates Anxiety-Like Behaviour. Journal of Biosciences, 43, 85-95. https://doi.org/10.1007/s12038-018-9732-y

[16] Adhikari, A., Lerner, T.N., Finkelstein, J., et al. (2015) Basomedial Amygdala Mediates Top-Down Control of Anxiety and Fear. Nature, 527, 179-185.

https://doi.org/10.1038/nature15698

[17] Tang, W.K., Lu, J.Y., Chen, Y.K., et al. (2011) Association of Frontal Subcortical Circuits Infarcts in Poststroke Depression: A Magnetic Resonance Imaging Study of 591 Chinese Patients with Ischemic Stroke. Journal of Geriatric Psychiatry and Neurology, 24, 44-49. https://doi.org/10.1177/0891988710392375

[18] Zhang, Y., Zhao, H., Fang, Y., Wang, S. and Zhou, H. (2017) The Association between Lesion Location, Sex and Poststroke Depression: Meta-Analysis. Brain and Behavior, 7, Article ID: e00788. https://doi.org/10.1002/brb3.788

[19] Schöttke, H. and Giabbiconi, C.M. (2015) Post-Stroke Depression and Post-Stroke Anxiety: Prevalence and Predictors. International Psychogeriatrics, 27, 1805-1812. https://doi.org/10.1017/S1041610215000988

[20] Mah, L., Szabuniewicz, C. and Fiocco, A.J. (2016) Can Anxiety Damage the Brain. Current Opinion in Psychiatry, 29, 56-63. https://doi.org/10.1097/YCO.0000000000000223

[21] Zhang, H.L., Guo, Z.N., Yang, G., et al. (2012) Compromised Cerebrovascular 
Modulation in Chronic Anxiety: Evidence from Cerebral Blood Flow Velocity Measured by Transcranial Doppler Sonography. Neuroscience Bulletin, 28, 723-728. https://doi.org/10.1007/s12264-012-1282-y 